\title{
A Scalable Model of the Substrate Network in Deep $N$-Well RF MOSFETs with Multiple Fingers
}

\author{
Jun Liu ${ }^{1,2}$, Marissa Condon ${ }^{2}$ \\ ${ }^{1}$ Key Laboratory of RF Circuits and Systems, Ministry of Education, Hangzhou Dianzi University, Hangzhou, China \\ ${ }^{2}$ School of Electronic Engineering, Dublin City University, Dublin, Ireland \\ E-mail: ljun77@163.com \\ Received January 18, 2011; revised March 4, 2011; accepted March 11, 2011
}

\begin{abstract}
A novel scalable model of substrate components for deep n-well (DNW) RF MOSFETs with different number of fingers is presented for the first time. The test structure developed in [1] is employed to directly access the characteristics of the substrate to extract the different substrate components. A methodology is developed to directly extract the parameters for the substrate network from the measured data. By using the measured two-port data of a set of nMOSFETs with different number of fingers, with the DNW in grounded and float configuration, respectively, the parameters of the scalable substrate model are obtained. The method and the substrate model are further verified and validated by matching the measured and simulated output admittances. Excellent agreement up to $40 \mathrm{GHz}$ for configurations in common-source has been achieved.
\end{abstract}

Keywords: Deep N-Well (DNW), RF Mosfets, Substrate Network, Scalable Model

\section{Introduction}

THE incorporation of a Deep N-Well (DNW) implantation into a standard CMOS technology has become a popular choice for reducing undesired interference in CMOS mixed-signal/RF SoC designs [2-6]. Substrate network parameters are of the utmost importance in accurately modeling the output admittance of RF MOSFETs. For mixed-signal/RF SoC design, a scalable model of RF MOSFETs is useful. Many papers have reported about scalable models of substrate network components [7-13]. However, there are few detailed works on scalable models with substrate network components in $D N W$ RF MOSFETs with different number of fingers. In contrast to the RF MOSFET without DNW implantation (as seen from the nMOSFETs in Figure 1), the $D N W$ actually partitions the substrate of a DNW RF MOSFET into three parts [1]: The $D N W$ itself, the p-well in the $D N W$, and the original substrate where the $D N W$ is formed. The $D N W$ layer forms a capacitive coupling path in the substrate, which exists no matter what the electrical configuration is. Furthermore, most previous works [7-19] dealt with substrate parasitic effects in RF MOSFETs by using resistance networks only. The capacitive coupling effect, which is physically in existence, is always neglected. All of these make the previously reported sub- strate models less physically reasonable to use for accurately extracting the substrate network components of DNW RF MOSFETs.

In this paper, a compact, physically based substrate network is proposed targeted specifically at $D N W$ RF MOSFET modeling. A novel test structure proposed in [1] is expanded and employed in deriving and extracting the $N_{f}$ - dependent equations involving substrate components in multi-finger DNW RF MOSFETs. The geometric effects such as shallow trench isolation (STI), which have never been considered in previous reported works, are accurately modeled. The results show that the substrate components within the p-well and the capacitances caused by the $D N W$ are strongly dependent on $N_{f}$, while the parasitic components in the original p-substrate have a slight dependence on $N_{f}$ in multi-finger devices.

To verify the validity of the derived scalable model of the substrate network components, a macro-model consisting of the BSIM3v3.2 model core with the proposed substrate-network based on the extracted parameters, is simulated in Agilent Advanced Design System (ADS). Excellent agreement between the simulated and measured output admittance for a set of devices with different number of fingers up to $40 \mathrm{GHz}$ validated the accuracy of the methodology proposed for DNW RF-MOSFET modeling in this paper. 


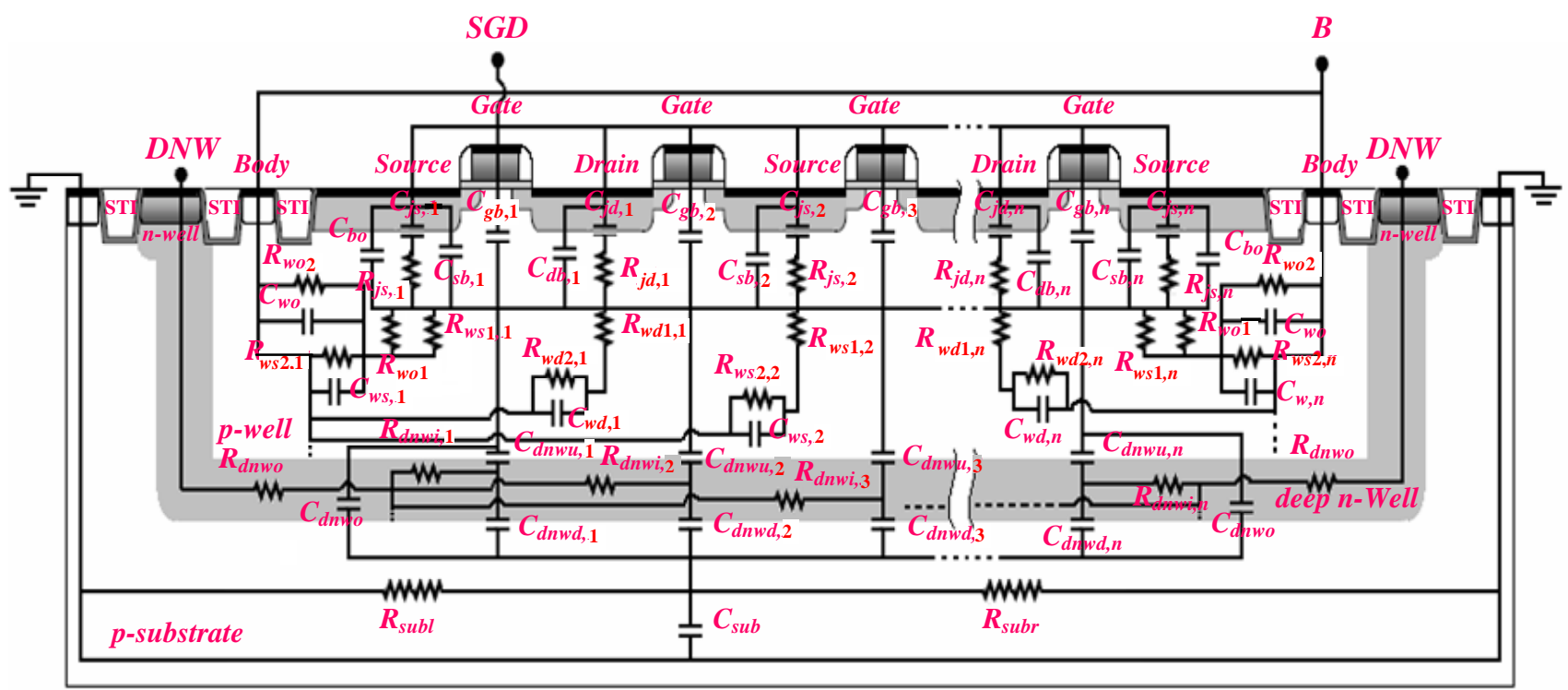

Figure 1. Equivalent circuit for the substrate resistance and capacitance networks of multi-finger (Nf) DNW RF MOSFET with all the source $(S)$, drain $(D)$ and gate $(G)$ terminals for different fingers connected together. Source, drain, and gate resistances are ignored for their slight contribution to the output impedance.

\section{Analysis of the Substrate Network and the Scalable Model Derivation}

A multi-finger DNW RF MOSFET with the test configuration proposed in [1] is investigated. All of the source $(S)$, drain $(D)$ and gate $(G)$ terminals for different fingers are connected together and used as port one, while the body $(B)$ terminal is port two, and the p-substrate is grounded, for two-port measurement. Figure 1 shows the substrate network when the junction diodes are turned off. In Figure 1, $C_{j s, i}, C_{j d, i}$ are each $S / D$ junction region capacitors, $R_{j s, i}, R_{j d, i}$ are each $S / D$ junction resistors. $C_{d n w o}$, which combined with $C_{w o}, C_{b o}, R_{w o 1}, R_{w o 2}$ and $R_{w o}$, is used to capture the difference between the inner and outer $S / D$ regions in this work. $C_{d n w u, i}$ and $C_{d n w d, i}$ represent the p-well-to- $D N W$ and the $D N W$-to-p-substrate capacitors under each finger region. $C_{w s, i}$ and $C_{w d, i}$ are each finger capacitors from the bottom of the $S / D$ regions to $B$ within the Deep $N$-Well. $R_{w s 1, i}, R_{w d 1, i}, R_{w s 2, i}$ and $R_{w d 2, i}$ represent the single finger resistors between the bottom of the $S / D$ region and $B$. $C_{s b, i}, C_{d b, i}$ and $C_{g b, i}$ are the $S$-to- $B, G$-to- $B$ and $D$-to- $B$ capacitors of each finger region, $R_{\text {subl }}, R_{\text {subr }}$ and $C_{\text {sub }}$ are the capacitor and the resistor of the p-substrate, $R_{d n w, i}$ represent the resistors of the DNW under each finger region. $R_{d n w o}$ represents the n-well ring resistor.

Based on the equivalent circuits identified in Figure 1, a simplified substrate network, as shown in Figure 2, with the following relationships can be obtained for any number of fingers:

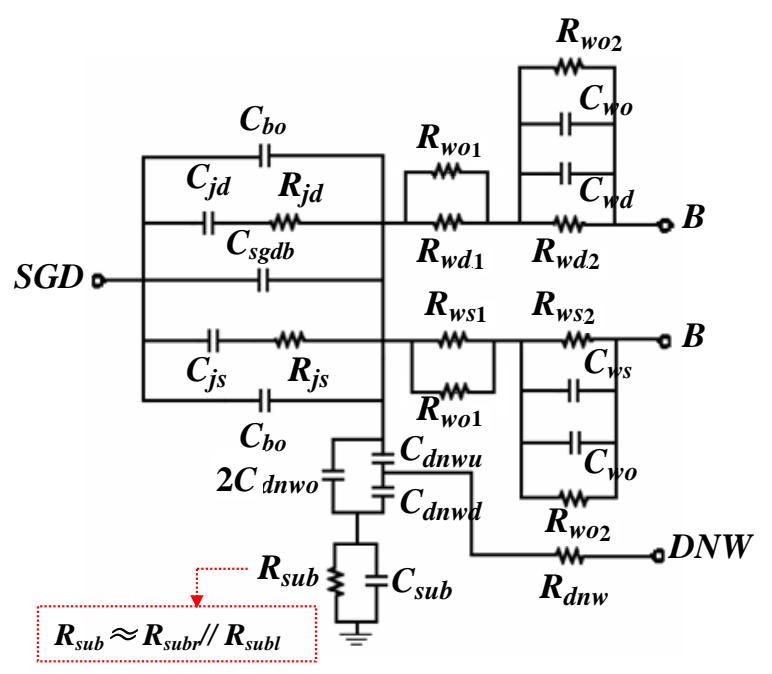

Figure 2. Equivalent circuit of multi-finger $D N W$ RF MOSFETs with $S / G / D$ terminals connected together.

$$
\begin{aligned}
& C_{j s / j d}=\sum_{i=1}^{N_{s / d}} C_{j s / j d, i} \\
& R_{j s / j d}^{-1}=\sum_{i=1}^{N_{s / d}} R_{j s / j d, i}^{-1} \\
& C_{s g d b}=\sum_{i=1}^{N_{f}}\left[C_{s b, i}+C_{g b, i}+C_{d b, i}\right] \\
& C_{w s / w d}=\sum_{i=1}^{N_{s / d}} C_{w s / w d, i}
\end{aligned}
$$




$$
\begin{aligned}
R_{w s 1 / w d 1}^{-1} & =\sum_{i=1}^{N_{s / d}} R_{w s 1 / w d 1, i}^{-1} \\
R_{\text {sub }}^{-1} & =R_{\text {subl }}^{-1}+R_{\text {subr }}^{-1} \\
R_{w s 2 / w d 2}^{-1} & =\sum_{i=1}^{N_{s / d}} R_{w s 2 / w d 2, i}^{-1} \\
C_{d n w u} & =\sum_{i=1}^{N_{f}}\left[C_{d n w u, i}\right] \\
C_{d n w d} & =\sum_{i=1}^{N_{f}}\left[C_{d n w d, i}\right] \\
R_{d n w}=2 R_{d n w o}+ & {\left[\sum_{i=1}^{N_{f}}\left[R_{d n w, i}^{-1}\right]\right]^{-1} }
\end{aligned}
$$

where, $C_{j s}, C_{j d}$ represent the total $S / D$ junction region capacitances, $R_{j s}, R_{j d}$ represent the total $S / D$ junction resistances, $R_{\text {sub }}$ represents the total resistance of the p-substrate, $C_{d n w}$ represents the total capacitance caused by the $D N W, C_{w s}, C_{w d}$ are the total capacitances from the bottom of the $S / D$ regions to $B$ within the Deep $N$-Well, $R_{w s 1 / w d 1}$ and $R_{w s 2 / w d 2}$ are the total resistances between the bottom of the $S / D$ regions and $B$ within the Deep $N$-Well and $N_{s}$ and $N_{d}$ represent the numbers of source and drain diffusion regions, respectively. In the model, when the number fingers is odd, $N_{s}=N_{d}=\left(N_{f}+1\right) / 2 . N_{s}=N_{f} / 2+1$ and $N_{d}=N_{f} / 2$ when the number of fingers is even.

Assuming that there are no differences in the inner $S / D$ regions, the above equations, (1a)-(1h), can be formed as follows:

$$
\begin{gathered}
C_{j s / j d}=N_{s / d} C_{j, i} \\
R_{j s / j d}=R_{j / i} / N_{s / d} \\
C_{s g d b}=N_{f}\left[2 C_{s d b, i}+C_{g b, i}\right] \\
C_{w s / w d}=N_{s / d} C_{w, i} \\
R_{w s 1 / w d 1}=\frac{R_{w 1, i}}{N_{s / d}}
\end{gathered}
$$

$$
\begin{gathered}
R_{w s 2 / w d 2}=\frac{R_{w 2, i}}{N_{s / d}} \\
C_{d n w u}=N_{f} C_{d n w u, i} \\
C_{d n w d}=N_{f} C_{d n w d, i} \\
R_{d n w}=2 R_{d n w o}+\frac{R_{d n w i}}{N_{f}}
\end{gathered}
$$

where

$$
\begin{gathered}
C_{j, i}=C_{j s, i}=C_{j d, i} R_{j, i}=R_{j s, i}=R_{j d, i}, C_{s d b}=C_{s b, i}=C_{d b, i} \\
C_{w, i}=C_{w s, i}=C_{w d, i}, R_{w 1, i}=R_{w s 1, i}=R_{w d 1, i}
\end{gathered}
$$

and

$$
R_{w 2, i}=R_{w s 2, i}=R_{w d 2, i} .
$$

In this paper, the following equations are used to empirically model the $N_{f}$ - dependence of $R_{\text {sub }}$ and $C_{\text {sub }}$ :

$$
\begin{gathered}
R_{\text {sub }}=R_{\text {subI }}+N_{f} R_{\text {subunit }} \\
C_{\text {sub }}=C_{\text {subI }}+N_{f} C_{\text {subunit }}
\end{gathered}
$$

where $R_{\text {subI }}$ and $C_{\text {subI }}$ represent the p-substrate resistance and capacitance of a one-finger device, $R_{\text {subunit }}$ and $C_{\text {subunit }}$ are used to explain the increase in $R_{\text {sub }}$ and $C_{\text {sub }}$ with the increase in the number of gate fingers.

\section{Scalable Model Parameter Extraction}

In order to accurately predict the scalability of the substrate elements, a direct parameter extraction methodology is of the utmost importance. In this work, two different test configurations are used. One has the $D N W$ floating (as shown in Figure 3(a)) and the other has the $D N W$ grounded (as shown in Figure 4(a)). In each case, all $S / D / G$ terminals for different fingers are connected together as port one, the $B$ terminal is port two, and the p-substrate grounded. The equivalent circuits shown in Figure 3(b) and Figure 4(b) can easily be derived from the complete equivalent circuit shown in Figure 2, for

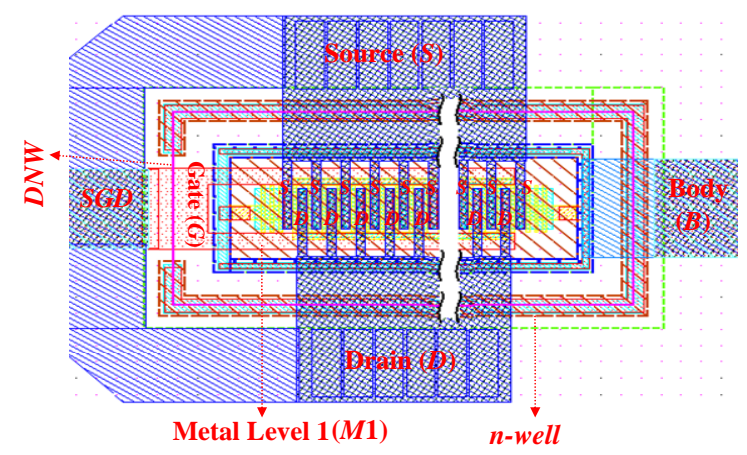

(a) 


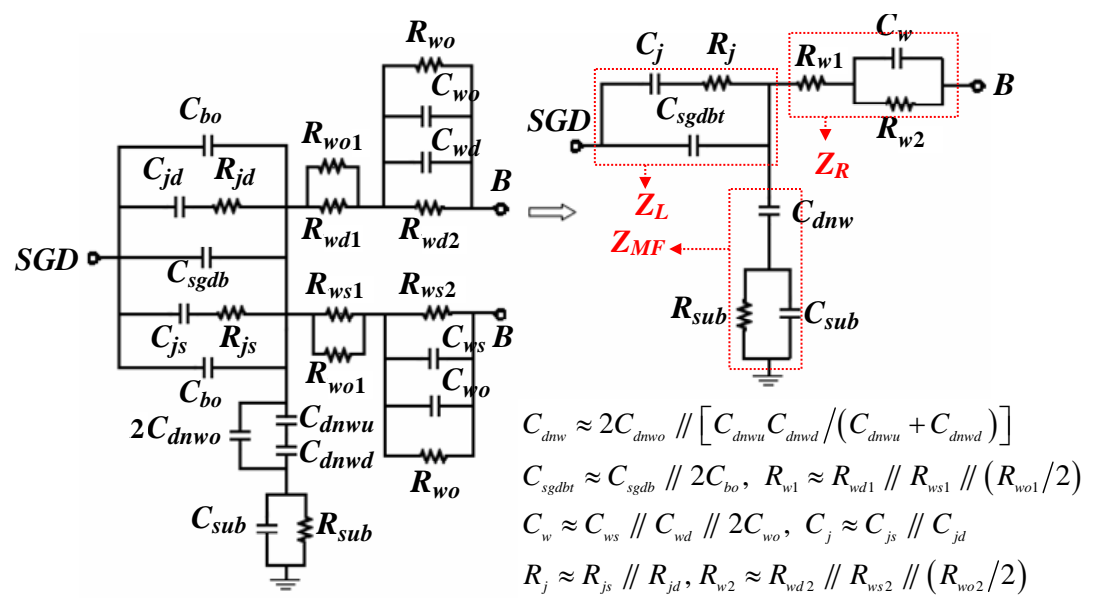

(b)

(c)

Figure 3. (a) Simplified layout plane figure of $D N W$ RF-MOSFETs with $S / G / D$ terminals connected together, while the $D N W$ is floating. (b) Equivalent circuit model for the device shown in Figure 3(a). $\boldsymbol{R}_{d n w}$ is ignored for its slight influence on two-port measurement. (c) Simplified equivalent circuit for parameter extraction.

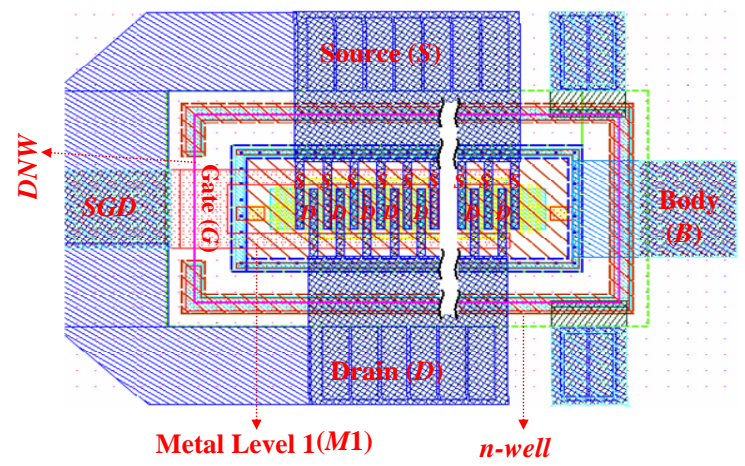

(a)

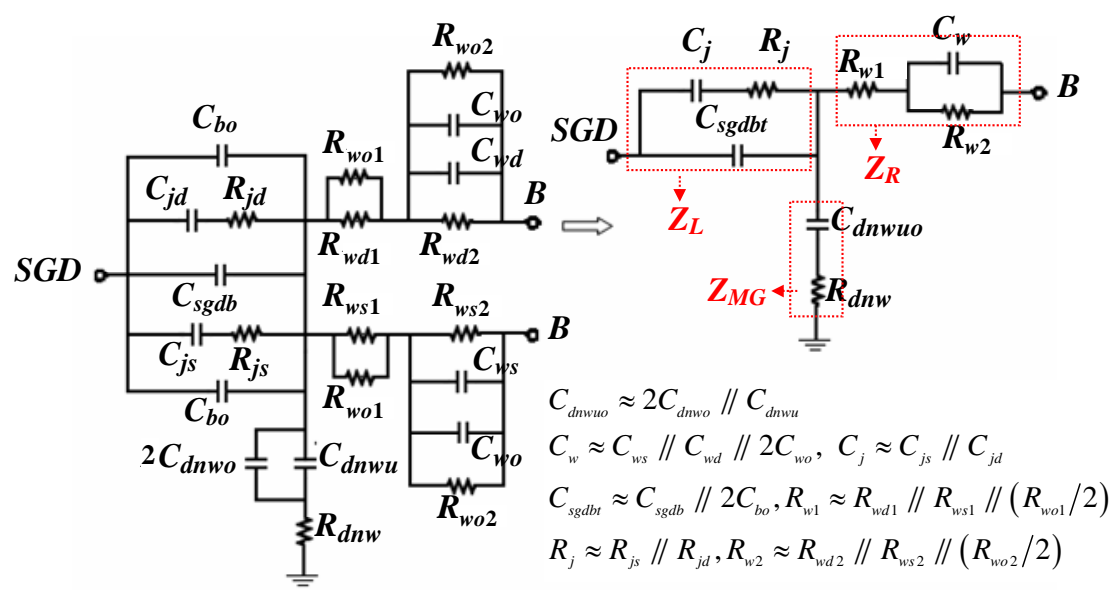

(b)

(c)

Figure 4. (a) Simplified layout plane figure of $D N W$ RF-MOSFETs with $S / G / D$ terminals connected together, with the $D N W$ grounded. (b) Equivalent circuit model for device shown in Figure 4(a). Since $\boldsymbol{R}_{d n w}$ is much smaller than $\boldsymbol{R}_{\text {sub }}$, the contribution of $C_{d n w d}, R_{s u b}$ and $C_{s u b}$ to $Z$-parameters becomes so slight that it can be ignored. (c) Simplified equivalent circuit for parameter extraction. 
As seen from Figure 3(b) and Figure 4(b), since the topologies from $S$ to $B$ are the same as that from $D$ to $B$, both of the equivalent circuits shown in Figure 3(b) and Figure 4(b) can be reduced to $T$-networks by using simple approaches as shown at the bottom of Figure 3(c) and Figure 4(c). Based on (2a)-(2i) and the approaches used to simplify Figure 3(b) and Figure 4(b) to Figure 3(c) and Figure 4(c), respectively, the elements of the two T-networks shown in Figure 3(c) and Figure 4(c) can be calculated with the following equations:

$$
\begin{gathered}
C_{j}=\left(N_{f}+1\right) C_{j, i} \\
R_{j}=R_{j, i} /\left(N_{f}+1\right) \\
C_{w}=2 C_{w o}+\left(N_{f}+1\right) C_{w, i} \\
R_{w 1}=\frac{0.5 R_{w o 1} R_{w 1, i} /\left(N_{f}+1\right)}{0.5 R_{w o 1}+R_{w 1, i} /\left(N_{f}+1\right)} \\
R_{w 2}=\frac{0.5 R_{w o 2} R_{w 2, i} /\left(N_{f}+1\right)}{0.5 R_{w o 2}+R_{w 2, i} /\left(N_{f}+1\right)} \\
C_{s g d b t}=2 C_{b o}+N_{f}\left[2 C_{s d b, i}+C_{g b, i}\right] \\
C_{d n w u o}=2 C_{d n w o}+N_{f} C_{d n w u, i} \\
C_{d n w}=2 C_{d n w o}+N_{f} C_{d n w, i}
\end{gathered}
$$

where

$$
C_{d n w, i}=\frac{C_{d n w u, i} C_{d n w d, i}}{C_{d n w u, i}+C_{d n w d, i}}
$$

Using (3h.1), $C_{d n w, i}$ can be calculated as follows:

$$
C_{d n w d, i}=\frac{C_{d n w u, i} C_{d n w, i}}{C_{d n w u, i}-C_{d n w, i}}
$$

(2c), (2g)-(2k) and (3a)-(3h) give the $N_{f}$-dependent equations of the equivalent circuit in Figure 2. This enables the direct identification of the scalability of the substrate components. This will be shown later in this section.

As the $Z_{L}$ and $Z_{R}$ of the $T$-network shown in Figure 4(c) are the same as the $Z_{L}$ and $Z_{R}$ shown in Figure 3(c), with the ground terminal as reference, the $Z$-parameters of the T-networks shown in Figure 3(c) and Figure 4(c) can be calculated approximately with the following equations:

$$
\begin{gathered}
{\left[Z_{L}\right]^{-1}=\left[Z_{d n w_{-} \text {floating }, 11}-Z_{d n w_{-} \text {floating }, 12}\right]^{-1}} \\
=\left[Z_{d n w_{-} \text {grounded }, 11}-Z_{d n w_{-} \text {grounded }, 12}\right]^{-1} \\
=\frac{\omega^{2} C_{j}^{2} R_{j}}{1+\omega^{2} C_{j}^{2} R_{j}^{2}}+j \frac{\omega C_{j}}{1+\omega^{2} C_{j}^{2} R_{j}^{2}}+j \omega C_{s g d b t}
\end{gathered}
$$

$$
\begin{aligned}
& Z_{R}=Z_{d n w_{-} \text {floating ,22 }}-Z_{d n w_{-} \text {floating }, 12} \\
& =Z_{d n w_{-} \text {grounded , 22 }}-Z_{d n w_{-} \text {grounded }, 12} \\
& =R_{w 1}+\frac{R_{w 2}}{1+\omega^{2} R_{w 2}^{2} C_{w}^{2}}-j \omega \frac{R_{w 2}^{2} C_{w}}{1+\omega^{2} R_{w 2}^{2} C_{w}^{2}} \\
& Z_{M F}=Z_{d n w_{-} \text {floating }, 12}=\frac{R_{\text {sub }}}{1+\omega^{2} R_{\text {sub }}^{2} C_{\text {sub }}^{2}} \\
& -j \omega \frac{R_{s u b}^{2} C_{s u b}}{1+\omega^{2} R_{s u b}^{2} C_{s u b}^{2}}-j \frac{1}{\omega C_{d n w}}
\end{aligned}
$$

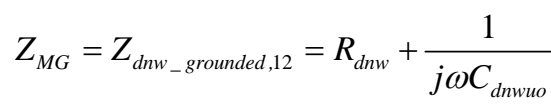

where $Z_{d n w \_f l o a t i n g}$ and $Z_{d n w \_ \text {grounded }}$ are measured $Z$-parameters of $D N W$ RF MOSFETs with $S / G / D$ terminals connected together, when the $D N W$ is floating or grounded, respectively.

Further, the real and imaginary parts of the above $Z$-parameter expressions can be rearranged as follows:

$$
\begin{gathered}
\frac{\omega^{2}}{\operatorname{Re}\left\{\left[Z_{L}\right]^{-1}\right\}}=\omega^{2} R_{j}+\frac{1}{C_{j}^{2} R_{j}} \\
C_{s g d b t}=\omega^{-1}\left\{\operatorname{Im}\left\{\left[Z_{L}\right]^{-1}\right\}-\frac{\omega C_{j}}{1+\omega^{2} C_{j}^{2} R_{j}^{2}}\right\} \\
-\frac{\omega}{\operatorname{Im}\left[Z_{R}\right]}=\omega^{2} C_{w}+\frac{1}{R_{w 2}^{2} C_{w}} \\
R_{w 1}=\operatorname{Re}\left[Z_{R}\right]-\frac{R_{w 2}}{1+\omega^{2} R_{w 2}^{2} C_{w}^{2}} \\
\left\{\operatorname{Re}\left[Z_{M F}\right]\right\}^{-1}=R_{\text {sub }}^{-1}+\omega^{2} R_{\text {sub }} C_{\text {sub }}^{2} \\
C_{d n w}=\left\{\omega\left[\operatorname{Im}\left[Z_{M F}\right]+\omega R_{\text {sub }}^{2} C_{\text {sub }} /\left(1+\omega^{2} R_{\text {sub }}^{2} C_{\text {sub }}^{2}\right)\right]\right\}^{-1}
\end{gathered}
$$

Using (5a) and (5c), $R_{\mathrm{j}}$ and $C_{w}$ can be extracted from the slopes of the linear regression curves of the experimental $\omega^{2} / \operatorname{Re}\left\{\left[Z_{L}\right]^{-1}\right\}$ and $-\omega / \operatorname{Im}\left[Z_{R}\right]$ versus $\omega^{2}$, respectively. (5a) and (5c), after subtracting $R_{j}$ and $C_{w}$, give $C_{j}$ and $R_{w 2}$. Further, (5b) and (5d) give $C_{s g d b t}$ and $R_{w 1}$. Using (5e), $R_{\text {sub }}$ and $C_{\text {sub }}$ can be determined from the intercept of the linear regression curve of the experimental $1 / \operatorname{Re}\left[Z_{M F}\right]$ versus $\omega^{2}$, and the slope gives $C_{\text {sub }}$ after subtracting $R_{\text {sub }}$. After subtracting $R_{\text {sub }}$ and $C_{\text {sub }}$, (5f) gives $C_{d n w}$. Using (5h), $C_{d n w u o}$ can be extracted from the slope of the linear regression curve of the experimental 
$\operatorname{Im}\left(Z_{M G}\right)$ versus $\omega$, while (5g) gives $R_{d n w}$ directly. Thus, all elements of the equivalent circuit of Figure 3(c) and/or Figure 4(c) are extracted.

For extracting the values of the derived scalable model parameters in (2c), (2g)-(2k) and (3a)-(3h), two different test structures for nine devices with different number of fingers $\left(N_{f}\right.$ of each device is $1,2,4,8,16,24,32,48$ and 64 , the length $\left(L_{f}\right)$ and width $\left(W_{f}\right)$ for each finger are fixed at $0.18 \mu \mathrm{m}$ and $2.5 \mu \mathrm{m}$ ), with the $D N W$ floating and grounded, respectively, were fabricated using the SMIC $0.18 \mu \mathrm{m}$ 1P6M RF-CMOS process. M1 is used to connect all of the $S / D / G$ terminals for different fingers together as port one, while the $B$ terminal is port two for two-port RF measurement.

In this work, two-port $S$-parameters were measured and de-embedded (Open + Short) for parasitics introduced by the GSG PAD using an Agilent E8363B Network Analyzer and a CASCADE Summit probe station. Then, the de-embedded $S$-parameters were transformed to $Z$-parameters for directly extracting all the parameters of the $T$-networks shown in Figure 3(c) and Figure 4(c) using the parameter extraction methodology developed in this section.

As mentioned in [1], when the junctions become significant, the equivalent circuit in Figure 2 and its corresponding parameter values are less reasonable. Thus, in this work, the extraction of the substrate network parameters is executed at $V_{B}=-1 \mathrm{~V}$ and $V_{S G D}=0 \mathrm{~V}$. A detailed extraction procedure for a 32-finger $D N W$ nMOSFET ( $L_{f}=0.18 \mu \mathrm{m}$ and $W_{f}=2.5 \mu \mathrm{m}$ for each finger) is given in Figure 5 to Figure 8. Excellent linear regressions validated the feasibility and accuracy of the parameter extraction methodology developed in this section. Similar extraction procedures are finally used for substrate parameter value extraction for the nine fabricated devices with different number of fingers at $V_{\mathrm{B}}=-1 \mathrm{~V}$ and $V_{\mathrm{SGD}}=0 \mathrm{~V}$. The extracted results are plotted in Figure 9.

\section{Scalable Model Verification and Validation}

Once $R_{j}, C_{j}, C_{w}, R_{w 1}, R_{w 2}, C_{s g d b}, R_{d n w}, R_{\text {sub }}, C_{s u b}, C_{d n w и o ~}$ and $C_{d n w}$ are extracted, by using (3a)-(3h) and (2i), $R_{j, i}, C_{j, i}$, $C_{w o}, C_{w, i}, R_{w 01}, R_{w 1, i}, R_{\mathrm{w} 02}, R_{w 2, i}, C_{b o},\left(2 C_{s d b i}+C_{g b i}\right)$, $R_{\text {dnwo }}, R_{d n w i}, R_{\text {subl }}, R_{\text {subunit }}, C_{\text {subl }}, C_{\text {subunit }}, C_{d n w o}, C_{d n w u i}$ and $C_{d n w i}$ can be obtained with a simple optimization procedure from the relationships between the total extracted results and $N_{f}$. After determining $C_{d n w u i}$ and $C_{d n w i}$, (3h.2) gives $C_{d n w d, i}$. Thus, (2a)-(2k) and (3a)-(3h) become only $N_{s / d}-$ and $N_{f}-$ dependence equations. Table 1 gives the extracted scalable model parameter values. Figure 9 depicts the comparisons between the extracted substrate resistances and capacitances of the nine DNW nMOSFETs and the modeled results based on the extracted parameter values shown in Table 1. The excellent agreement between the extracted and modeled $N_{f}$ - dependent substrate network components verifies that the proposed scalable model ((3a-3h)) can accurately describe the scalabilities of the substrate network components of $D N W$ MOSFETs.

To verify the validity of the proposed substrate network, the accuracy of the derived scalable model and the developed methodology for parameter extraction, multi-finger $D N W$ nMOSFETs, with the $G$ terminal defining port one, the $D$ terminal defining port two and the $S, B$ and the p-substrate connected together with ground serving as the common terminal (i.e. common-source test configuration) with the $D N W$ connected to ground, are also fabricated and tested. A macro-model (as shown in

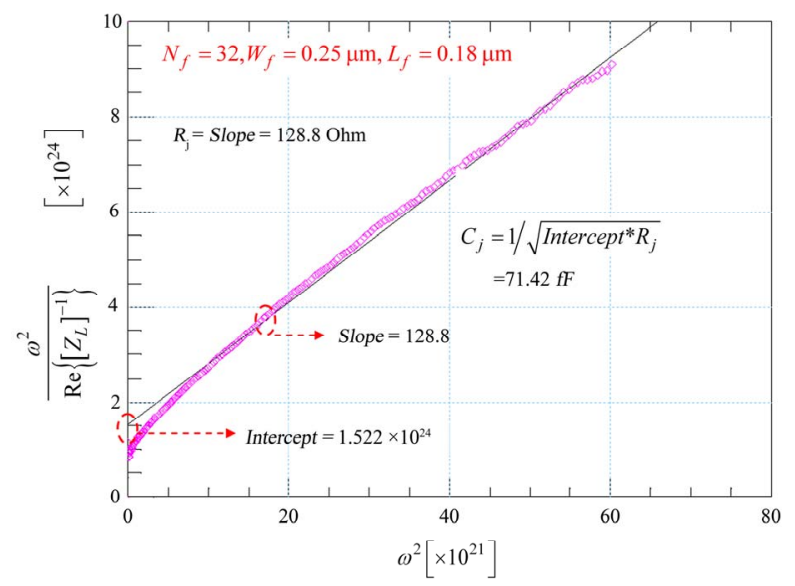

(a)

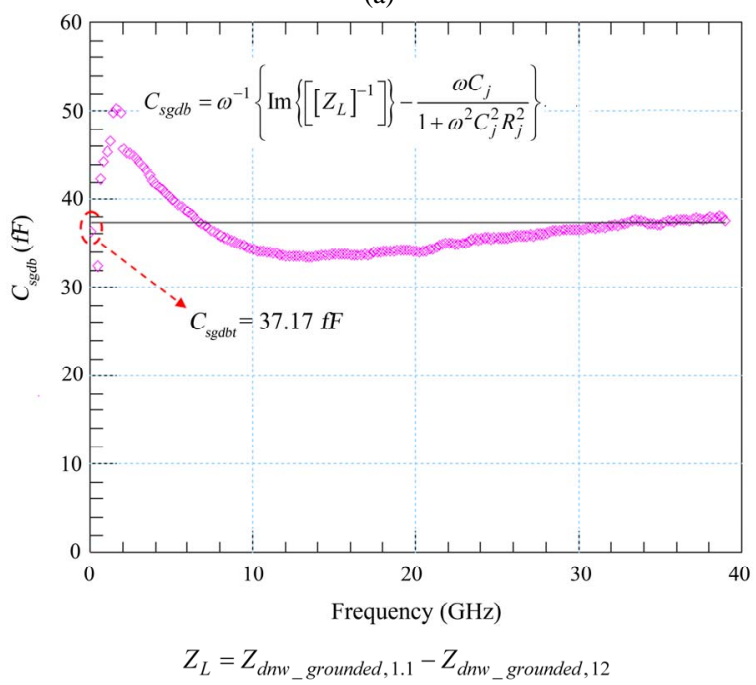

(b)

Figure 5. (a) Determine $\boldsymbol{R}_{j}$ from the slope of the linear regression curve of the experimental $w^{2} / \operatorname{Re}\left\{\left[Z_{L}\right]-1\right\}$ versus $\omega^{2} . C_{j}$ can be calculated from the intercept. (b) After subtracting $R_{j}$ and $C_{j}$, (5b) gives $C_{\text {sgdb }}$. 


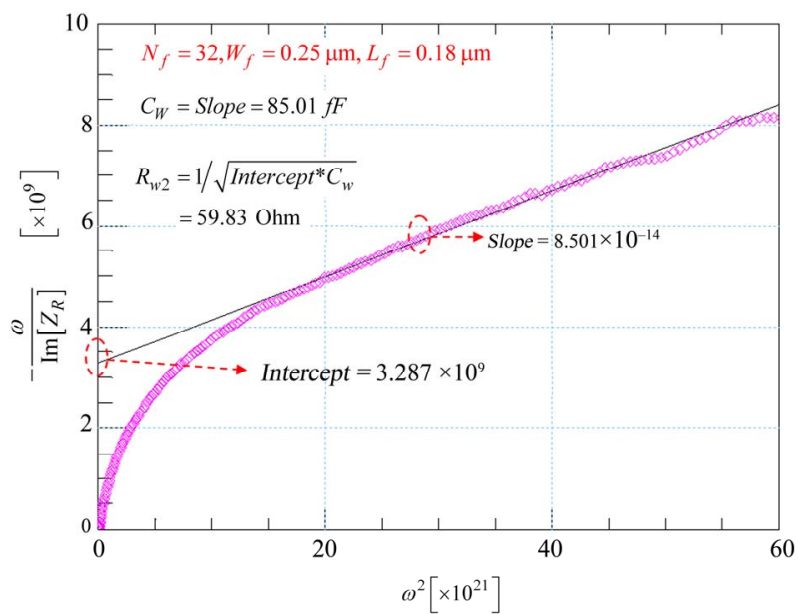

(a)

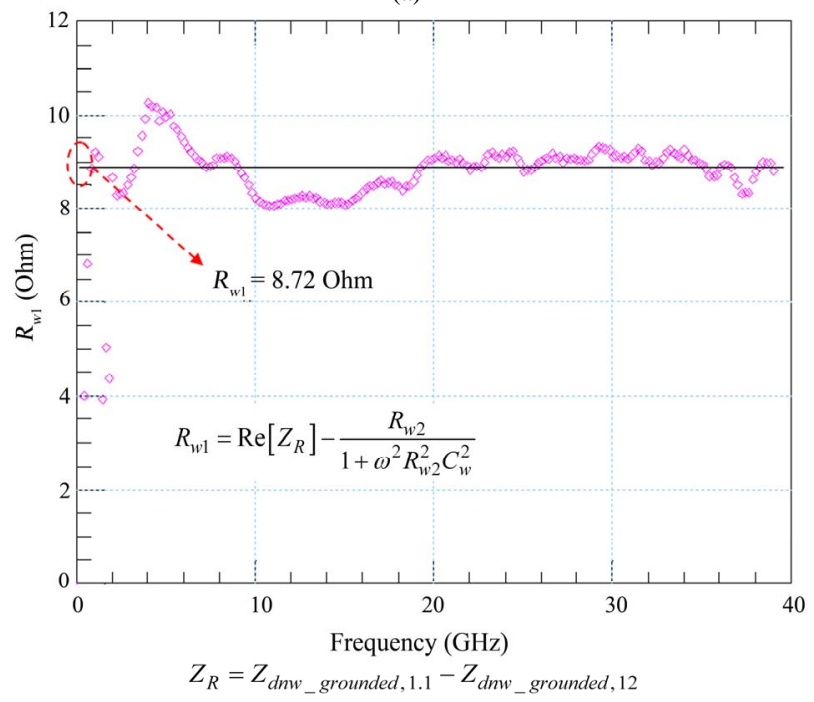

(b)

Figure 6. (a) Extract $C_{w}$ from the slope of the linear regression curve of the experimental $-w / \operatorname{Im}[Z R]$ versus $w^{2} \cdot \boldsymbol{R}_{w 2}$ can be extracted from the intercept. (b) After subtracting $R_{w 2}$ and $C_{w}$, (5d) gives $R_{w 1}$.

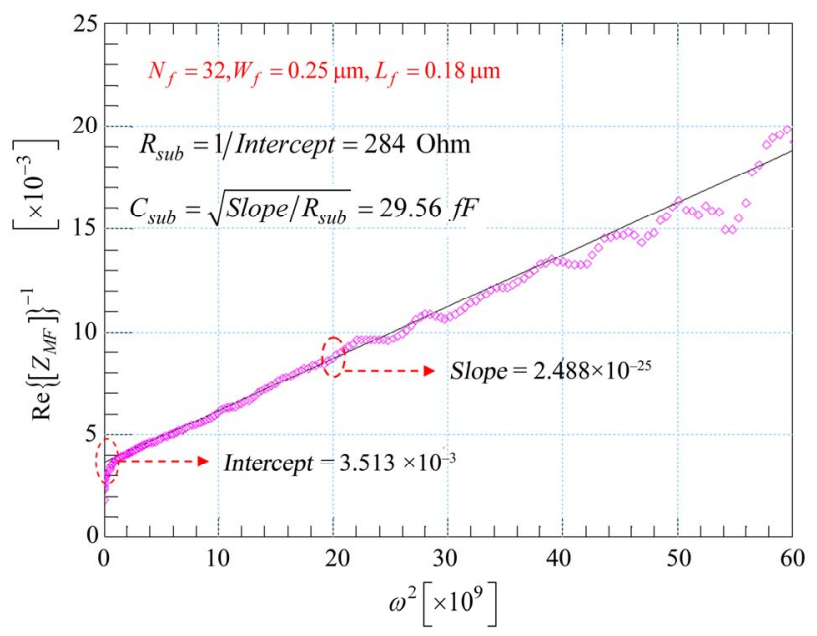

(a)

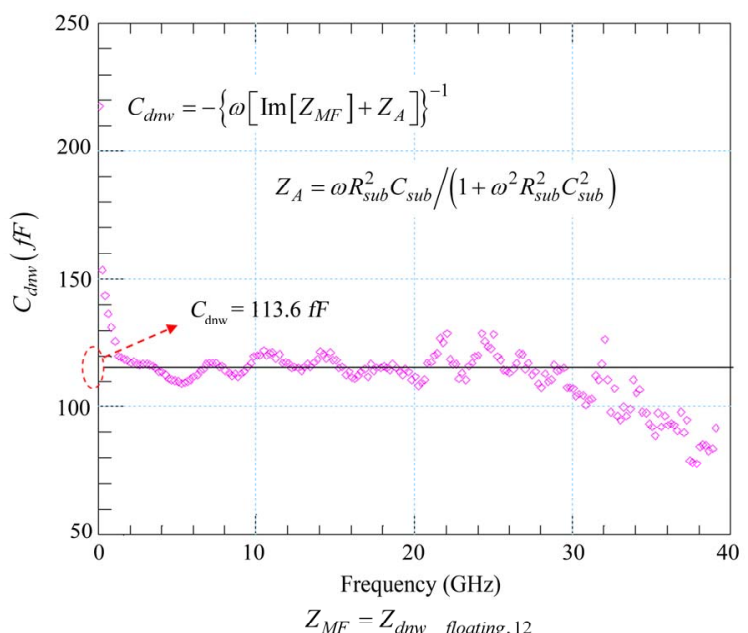

(b)

Figure 7. (a) Extract $\boldsymbol{R}_{\text {sub }}$ from the intercept of the experimental $\left\{\operatorname{Re}\left[Z_{M F}\right]\right\}-1$ versus $w^{2}$, and the slope gives $C_{s u b}$ after subtracting $R_{s u b}$. (b) After subtracting $R_{\text {sub }}$ and $C_{\text {sub }}$, (5f) gives $C_{d n w}$.

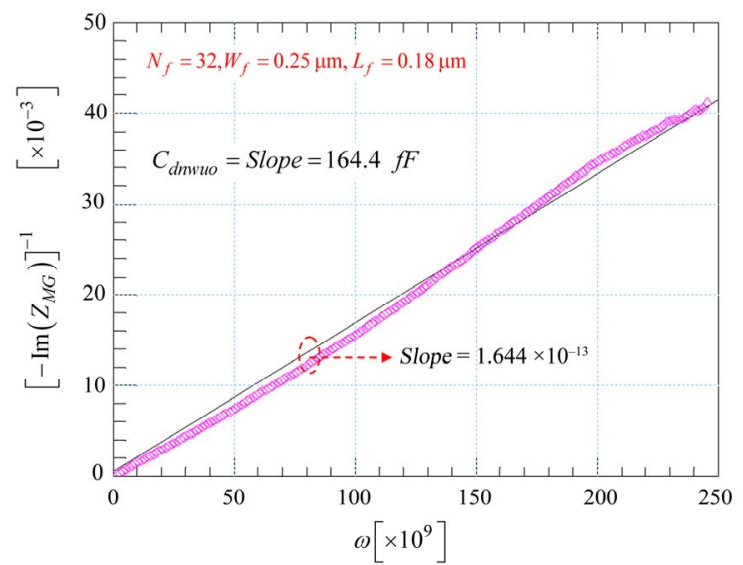

(a)

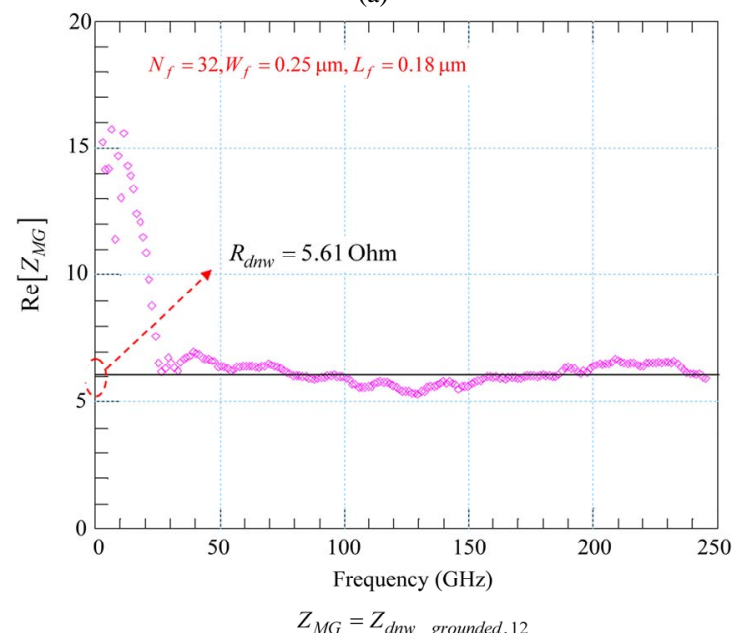

(b)

Figure 8. (a) Extract $C_{d n w u o}$ from the slope of the linear regression curve of the experimental $\operatorname{Im}\left[Z_{M G}\right]$ versus $w$. (b) $\boldsymbol{R}_{d n w}$ can be determined from the real part of $Z_{M G}$. 
Figure 10) for common-source connected $D N W$ RF MOSFETs modeling was developed. The model consists of the BSIM3v3.2 model core with the proposed new substrate-network and is simulated in Agilent Advanced Design System (ADS) directly.

In Figure 10, $R_{g}, R_{d}$, and $R_{S}$ are $G, D$ and $S$ terminal series resistances, $C_{d s}$ is $D$-to- $S$ capacitance. $C_{g s}$, and $C_{g d}$ represent the $G$-to- $S$ and $G$-to- $D$ capacitances, respectively. $C_{g b}, C_{d b}$ and $C_{s b}$ indicate the $G$-to- $B, D$-to-B, $S$-to- $B$ capacitances, and the sum of the three components has been extracted in section 4 . A conventional method developed in [13] is used to extract the initial values of three terminal series resistances from de-em-

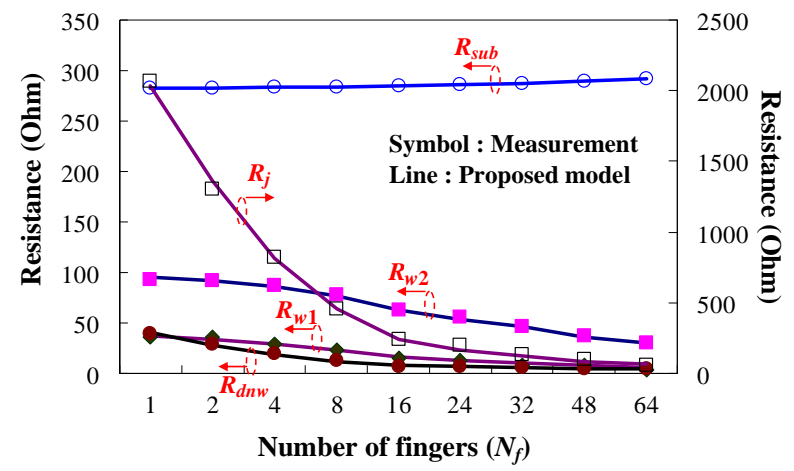

(a)

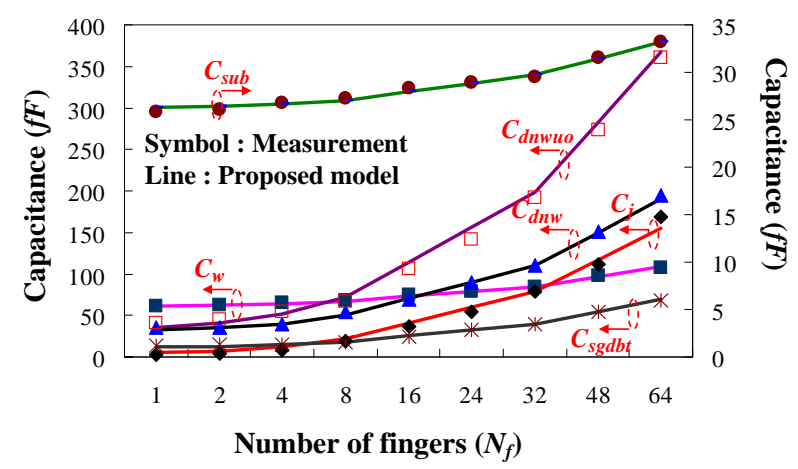

(b)

Figure 9. (a) Extracted and modeled substrate resistances and (b) capacitances of DNW nMOSFETs with different number of fingers, while the length $\left(L_{f}\right)$ and width $\left(W_{f}\right)$ for each finger are fixed at $0.18 \mu \mathrm{m}$ and $2.5 \mu \mathrm{m}$.

Table 1. Extracted parameter values of the proposed model of the substrate network in DNW RF MOSFETs

\begin{tabular}{ccccc}
\hline$R_{j, i}(\Omega)$ & $C_{j, i}(f F)$ & $C_{\text {wo }}(f F)$ & $C_{w, i}(f F)$ & $R_{\text {wo } 1}(\Omega)$ \\
\hline 4162 & 2.395 & 30.64 & 0.737 & 87.78 \\
$R_{w o 2}(\Omega)$ & $R_{w 2, i}(\Omega)$ & $C_{b o}(f F)$ & \multicolumn{2}{c}{$2 C_{\text {sdbi }}+C_{g b i}(f F)$} \\
203.7 & 2775 & 5.471 & \multicolumn{2}{c}{0.91} \\
$R_{\text {dnwi }}(\Omega)$ & $R_{\text {subl }}(\Omega)$ & $R_{\text {subunit }}(\Omega)$ & $C_{\text {subl }}(f F)$ & $C_{\text {subunit }}(f F)$ \\
73.79 & 282.8 & 0.137 & 26.18 & 0.11 \\
$C_{\text {dnwui }}(f F)$ & $C_{\text {dnwdi }}(f F)$ & $R_{w 1, i}(\Omega)$ & $R_{\text {dnwo }}(\Omega)$ & $C_{\text {dnwo }}(f F)$ \\
5.045 & 4.771 & 447.7 & 3.46 & 15.2 \\
\hline
\end{tabular}

Table 2. Values of the extracted external capacitors from common source connected devices with different $N_{f}$, at zero bias. $\left(L_{f}=0.18 \mu \mathrm{m} ; W_{f}=2.5 \mu \mathrm{m}\right)$

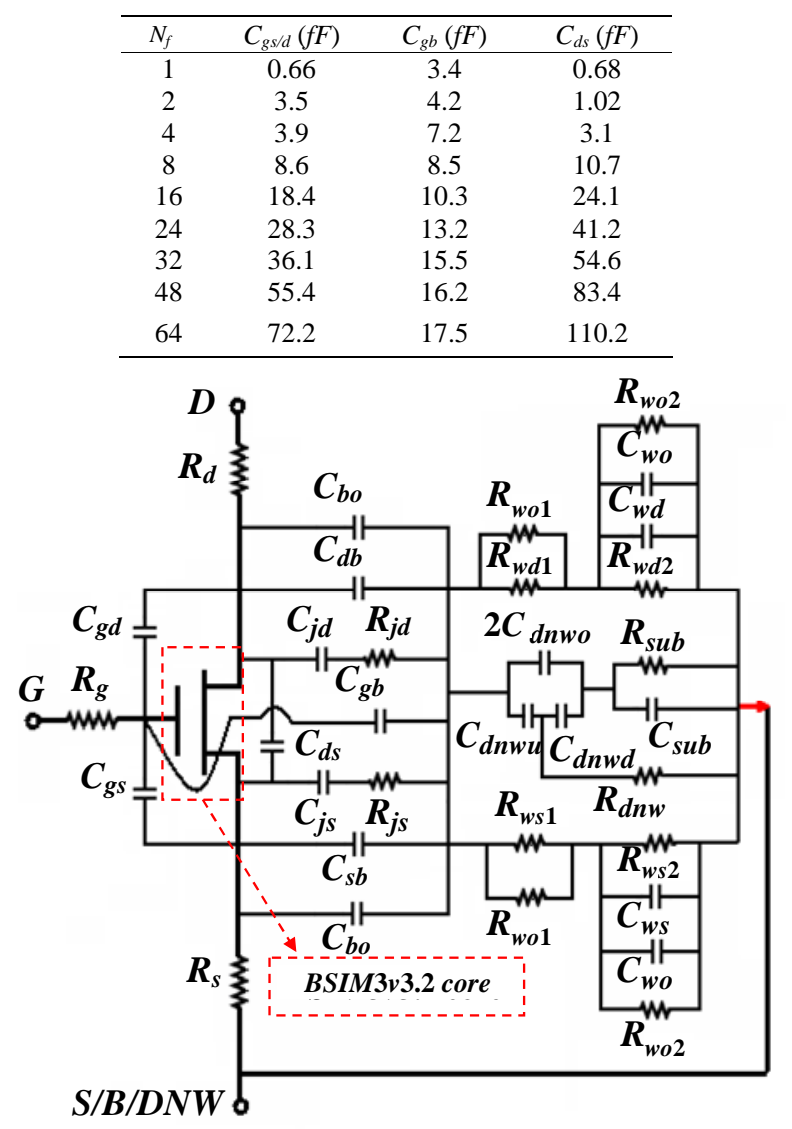

Figure 10. Macro-model for DNW RF-MOSFETs modeling when $S / D$ junctions are not significant. The test configuration with the $G$ terminal defining port one, the $D$ terminal defining port two and the $S, B$ and the p-substrate connected together with ground serving as the common terminal (i.e. common-source test configuration), with the $D N W$ is tied to ground using M1 (metal level 1), are used in two-port measurement. All the parameters of the BSIM3v3.2, including the terminal resistances $R_{d}, R_{g}$ and $R_{s}$, are extracted beforehand.

bedded $Y$-parameters. By using the extraction method proposed in [13], the following equations are employed for the remaining components extraction:

$$
\begin{gathered}
C_{g d}=\frac{\operatorname{Im}\left(Y_{12}\right)}{\omega} \\
C_{g s}=C_{g d} \\
C_{g b}=\frac{\operatorname{Im}\left(Y_{11}+Y_{12}\right)}{\omega}-C_{g d}
\end{gathered}
$$

According to (1c), the total $C_{g b}$ of an RF MOSFET with the number of fingers is $N_{f}$ can be calculated as follows: 


$$
C_{g b}=N_{f} C_{g b, i}
$$

Thus, $C_{g b, i}$ can be extracted for two or more devices with different number of fingers. Once $C_{g b, i}$ is obtained, (3f) gives $C_{s d b, i}$.

$$
C_{s d b, i}=\frac{\left[C_{s g d b t}-2 C_{b o}-N_{f} C_{g b, i}\right]}{2 N_{f}}
$$

In this work, the extracted values of $C_{g b, i}$ and $C_{s d b, i}$ for multi-finger devices with the length $(L)$ and width $(W)$ for each finger fixed at $0.18 \mu \mathrm{m}$ and $2.5 \mu \mathrm{m}$, are $0.338 \mathrm{fF}$ and $0.285 \mathrm{fF}$, respectively. $C_{d s}$ in Figure $\mathbf{1 0}$ is calculated from de-embedded $Y$-parameters of the common-source connected nMOSFET as follows:

$$
C_{d s}=\frac{\operatorname{Im}\left(Y_{22}-Y_{12}\right)}{\omega}-\frac{C_{d}\left(C_{s}+C_{t}\right)}{C_{d}+C_{s}+C_{t}}
$$

where

$$
\begin{gathered}
C_{d}=C_{j d}+C_{b o}+C_{d b}, \quad C_{s}=C_{j s}+C_{b o}+C_{s b}, \\
C_{t}=2 C_{w o}+C_{w d}+C_{w s}+\frac{C_{n} C_{s u b}}{C_{n}+C_{s u b}}
\end{gathered}
$$

and

$$
C_{n}=2 C_{d n w o}+\frac{C_{d n w u} C_{d n w d}}{C_{d n w u}+C_{d n w d}} .
$$

The external capacitances in Figure 10 (i.e. $C_{g d}, C_{g s}$ and $C_{d s}$ ) extracted from the nine devices with different $N_{f}$ at zero-bias condition $\left(V_{G}=0 \mathrm{~V} ; V_{D}=0 \mathrm{~V}\right.$ and $V_{S / B / D N W}=$ $0 \mathrm{~V}$ ) are listed in Table 2. After all the parameters have been extracted, measured and simulated output admittances $\left(Y_{22}\right)$ at zero-bias for the nine devices with different number of fingers are compared and plotted in Figure 11. Excellent agreement is achieved between the measured and simulated results. Due to the oscillation of the measurements at high frequencies, the resistive parasitics of the substrate are hard to be extracted accurately, which is further introducing errors between the measured and simulated results of the real parts of the output admittances of transistors.

\section{Summary}

A compact, physically based scalable model for the substrate network of DNW RF MOSFETs has been demonstrated. All of the substrate components are directly extracted from two-port measurements. The derived and extracted scalable model is directly used to capture the substrate characteristics of common-source connected devices. The model shows excellent agreement with measured output admittances of devices with different number of fingers at an operation frequency up to $40 \mathrm{GHz}$. The model and methodology developed in this paper also can be used to accurately extract the substrate network in RF MOSFETs without $D N W$ implantation by removing the

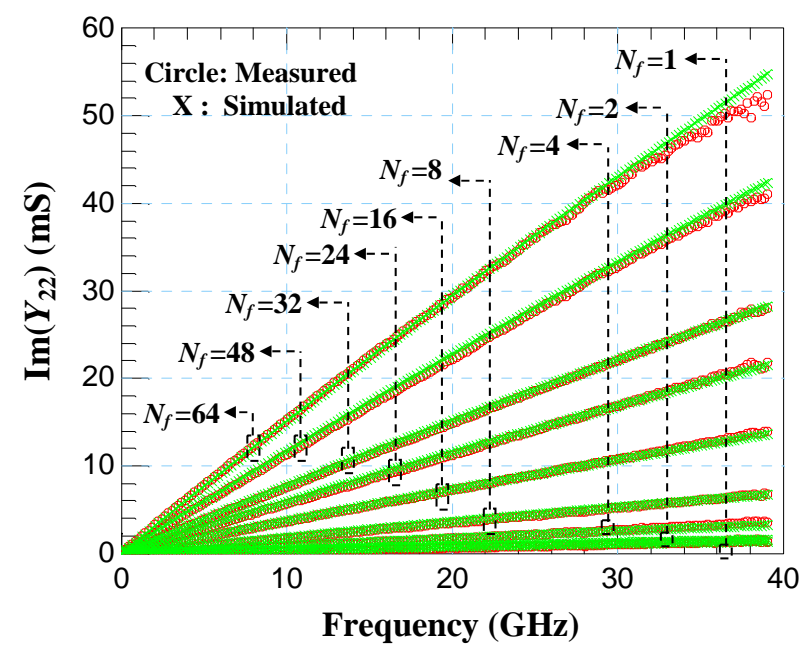

(a)

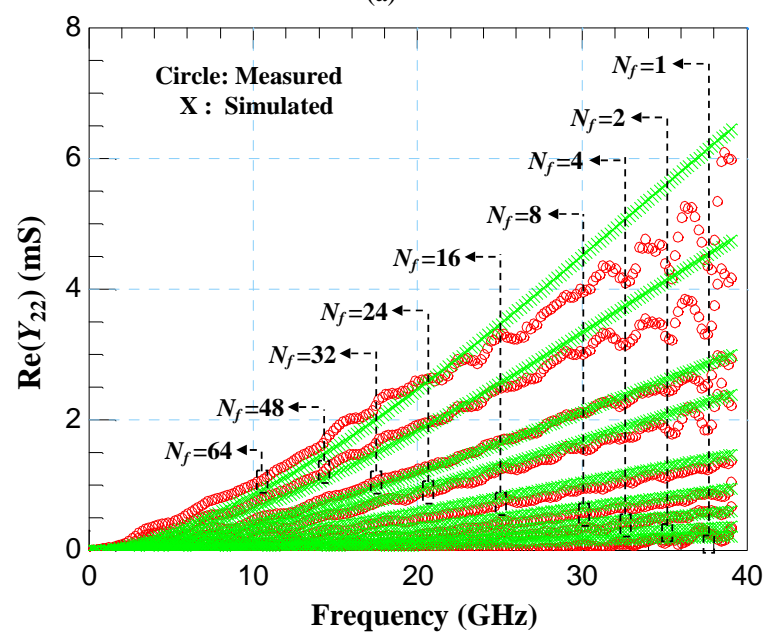

(b)

Figure 11. Measured and simulated output admittances of $D N W$ nMOSFETs with different number of fingers at zero bias $\left(V_{G}=0 \mathrm{~V}, V_{D}=0 \mathrm{~V}\right.$ and $\left.V_{S / B / D N W}=0 \mathrm{~V}\right)$. All the devices are connected in common source configuration, while the $D N W$ is grounded.

sub-network for the DNW.

\section{References}

[1] J. Liu, L. L. Sun, L. L. Lou, H. Wang and C. McCorkell, "A Simple Test Structure for Directly Extracting Substrate Network Components in Deep $N$-Well RF CMOS Modeling,” IEEE Electron Device Letters, Vol. 30, No. 11, 2009, pp. 1200-1202.

[2] J. G. Su, H. M. Hsu, S. C. Wong, C. Y. Chang, T. Y. Huang and J. Y. C. Sun, "Improving the RF Performance of 0.18-um CMOS with Deep $N$-Well Implantation," IEEE Electron Device Letters, Vol. 22, No. 10, 2001, pp. 481-483.

[3] K. W. Chew, J. Zhang, K. Shao, W. B. Loh, and S. F. Chu, "Impact of Deep $N$-Well Implantation on Substrate Noise Coupling and RF Transistor Performance for Sys- 
tems-on-a-Chip Integration," Proceeding of the 32nd European Solid-State Device Research Conference, Bologna, 24-26 September 2002, pp. 251-254.

[4] D. Kosaka, M. Nagata, Y. Hiraoka, I. Imanishi, M. Maeda, Y. Murasaka and A. Iwata, "Isolation Strategy Against Substrate Coupling in CMOS Mixed-Signal/RF Circuits," Symposium on VLSI Circuits Digest of Technical Papers, Kyoto, 16-18 June 2005, pp. 276-279.

[5] J. Kang, D. Yu, Y. Yang and B. Kim, "Highly Linear 0.18-m CMOS Power Amplifier with Deep-N-Well Structure,” IEEE Journal of Solid-State Circuits, Vol. 41, No. 5, 2006, pp. 1073-1080. doi:10.1109/JSSC.2006.874059

[6] S. F. W. M. Hatta and N. Soin, "Performance of the Forward-Biased RF LNA with Deep $N$-Well NMOS Transistor," Proceeding of International Conference on Semiconductor Electronics, Johor Bahru, 25-27 November 2008, pp. 465-469.

[7] J. Han and H. Shin, "A Scalable Model for the Substrate Resistance in Multi-Finger RF MOSFETs,” IEEE MTT-S International Microwave Symposium Digest, Philadelphia, 8-13 June 2003, pp. 2105-2108.

[8] Y. Cheng and M. Matloubian, "Parameter Extraction of Accurate and Scaleable Substrate Resistance Components in RF MOSFETs," IEEE Electron Device Letters, Vol. 23, No. 4, 2002, pp. 221-223. doi:10.1109/55.992845

[9] N. Srirattana, D. Heo, H. M. Park, A. Raghavan, P. E. Allen and J. Laskar, "A New Analytical Scalable Substrate Network Model for RF MOSFETs," IEEE MTT-S Microwave Symposium Digest, Fort Worth, 6-11 June 2004, pp. 699-702.

[10] I. M. Kang, S. J. Jung, T. H. Choi, H. W. Lee, G. Jo, Y. K. Kim, H. G. Kim and K. M. Choi, "Scalable Model of Substrate Resistance Components in RF MOSFETs with Bar-Type Body Contact Considered Layout Dimensions,” IEEE Electron Device Letters, Vol. 30, No. 4, 2009, pp. 404-406. doi:10.1109/LED.2009.2014085

[11] S. P. Voinigescu, M. Tazlauanu, P. C. Ho and M. T. Yang, "Direct Extraction Methodology for Geometry-Scalable RF-CMOS Models,” International Conference on Microelectronic Test Structures, Awaji, 22-25 March 2004, pp. 235-240.
[12] S. P. Kao, C. Y. Lee, C. Y. Wang, J. D.-S. Deng, C. C. Chang and C. H. Kao, “An Analytical Extraction Method for Scalable Substrate Resistance Model in RF MOSFETs,” 2007 International Semiconductor Device Research Symposium, College Park, 12-14 December 2007, pp. 1-2.

[13] B. Parvais, S. Hu, M. Dehan, A. Mercha and S. Decoutere, "An Analytical Extraction Method for Scalable Substrate Resistance Model in RF MOSFETs,” Custom Integrated Circuits Conference, San Jose, 16-19 September 2007, pp. 503-506.

[14] Y. Cheng, M. J. Deen and C. H. Chen, "MOSFET Modeling for RF IC Design,” IEEE Transactions on Electron Devices, Vol. 52, No. 7, 2005, pp. 1286-1303. doi:10.1109/TED.2005.850656

[15] M. M. Tabrizi, E. Fathi, M. Fathipour and N. Masoumi, "Extracting of Substrate Network Resistances in RF CMOS Transistors,” Topical Meeting on Silicon Monolithic Integrated Circuits in RF Systems, Atlanta, 8-10 September 2004, pp. 219-222.

[16] J. Han, M. Je and H. Shin, "A Simple and Accurate Method for Extracting Substrate Resistance of RF MOSFETs,” IEEE Electron Device Letters, Vol. 23, No. 7, 2002, pp. 434-436.

[17] Y. S. Lin, “An Analysis of Small-Signal Source-Body Resistance Effect on RF MOSFET for Low-Cost System-on-Chip (SoC) Applications," IEEE Transactions on Electron Devices, Vol. 52, No. 7, 2005, pp. 1442-1451. doi:10.1109/TED.2005.850691

[18] S. C. Rustagi, L. Huailin, S. Jinglin and Z. X. Yong, "BSIM3 RF Models for MOS Transistors: A Novel Technique for Substrate Network Extraction,” Proceeding of IEEE International Conference on Microelectronic Test Structures, Monterey, 17-20 March 2003, pp. 118-123.

[19] U. Mahalingam, S. C. Rustagi and G. S. Samudra, "Direct Extraction of Substrate Network Parameters for RF MOSFET Modeling Using a Simple Test Structure," IEEE Device Letters, Vol. 27, No. 2, 2006, pp. 130-132. doi:10.1109/LED.2005.863132 